\title{
Discrete-Time Langevin Motion in a Gibbs Potential
}

\author{
Reza Rastegar', Alexander Roitershtein ${ }^{1}$, Vadim Roytershteyn ${ }^{2}$, Jiyeon Suh $^{3}$ \\ ${ }^{1}$ Department of Mathematics, Iowa State University, Ames, USA \\ ${ }^{2}$ Department of Electrical and Computer Engineering, University of California San Diego, San Diego, USA \\ ${ }^{3}$ Department of Mathematics, Grand Valley State University, Allendale, USA \\ Email: rastegar@iastate.edu, roiterst@iastate.edu, roytersh@gmail.com, suhj@gvsu.edu
}

Received September 8, 2012; revised October 8, 2012; accepted October 15, 2012

\begin{abstract}
We consider a multivariate Langevin equation in discrete time, driven by a force induced by certain Gibbs' states. The main goal of the paper is to study the asymptotic behavior of a random walk with stationary increments (which are interpreted as discrete-time speed terms) satisfying the Langevin equation. We observe that (stable) functional limit theorems and laws of iterated logarithm for regular random walks with i.i.d. heavy-tailed increments can be carried over to the motion of the Langevin particle.
\end{abstract}

Keywords: Langevin Equation; Dynamics of a Moving Particle; Multivariate Regular Variation; Chains with Complete Connections

\section{Introduction}

We start with the following equation describing a discrete-time motion in $\mathbb{R}^{\mathrm{d}}, \mathrm{d} \geq 1$, of a particle with mass $m$ in the presence of a random potential and a viscosity force proportional to velocity:

$$
m\left(V_{n}-V_{n-1}\right)=-\Gamma V_{n-1}+F_{n}, n \in \mathbb{N} .
$$

Here d-vector $V_{n}$ is the velocity at time $n, \mathrm{~d} \times \mathrm{d}$ matrix $\Gamma$ represents an anisotropic damping coefficient, and d-vector $F_{n}$ is a random force applied at time $n$. The above equation is a discrete-time counterpart of the Langevin SDE $\mathrm{d} V_{t}=-\Gamma V_{t}+\mathrm{d} W_{t} \quad[1,2]$. Applications of the Langevin equation with a random non-Gaussian term $W_{t}$ are addressed, for instance, in $[3,4]$. Setting $M=I-m^{-1} \Gamma$ and $Q_{n}=m^{-1} F_{n}$, we obtain:

$$
V_{n}=M V_{n-1}+Q_{n}, n \in \mathbb{N} \text {. }
$$

The random walk $\left(X_{n}\right)_{n \geq 0}$ associated with this equation is given by

$$
X_{n}=X_{0}+\sum_{k=1}^{n} V_{k}, n \in \mathbb{N} .
$$

Similar models of random motion in dimension one, with i.i.d. forces $\left(Q_{n}\right)_{n \in \mathbb{Z}}$ were considered in [5-8], see also $[9,10]$ and references therein. See, for instance, [11-14] for interesting examples of applications of Equation (1) with i.d.d. coefficients in various areas.

In this paper we will assume that the coefficients $\left(Q_{n}\right)_{n \in \mathbb{Z}}$ are induced (in the sense of the following definition) by certain Gibbs's states.
Definition 1. Coefficients $\left(Q_{n}\right)_{n \in \mathbb{Z}}$ are said to be induced by random variables $\left(Z_{n}\right)_{n \in \mathbb{Z}}$, each valued in a finite set $D$, if there exists a sequence of independent random d-vectors $Q_{n}=\left(Q_{n, i}\right)_{n \in \mathbb{Z}, i \in D}$ which is independent of $\left(Z_{n}\right)_{n \in \mathbb{Z}}$ and is such that for a fixed $i \in D, Q_{n}=\left(Q_{n, i}\right)_{n \in \mathbb{Z}}$ are i.i.d. and $Q_{n}=Q_{n, Z_{n}}$.

The randomness of $\left(Q_{n}\right)_{n \in \mathbb{Z}}$ is due to two factors:

1) Random environment $\left(Z_{n}\right)_{n \in \mathbb{Z}}$ which describes a "state of Nature"; and, given the realization of $\left(Z_{n}\right)_{n \in \mathbb{Z}}$,

2) The "intrinsic" randomness of systems' characteristics which is captured by the random variables $Q_{n}=Q_{n, Z_{n}}$.

Note that when $\left(Z_{n}\right)_{n \in \mathbb{Z}}$ is a finite Markov chain, $Q_{n}=Q_{n, Z_{n}}$ is a Hidden Markov Model. See, for instance, [15] for a survey of HMM and their applications. Heavy tailed HMM as random coefficients of multivariate linear time-series models have been considered, for instance, in $[16,17]$. In the context of financial time series, $Z_{n}$ can be interpreted as an exogenous factor determined by the current state of the underlying economy. The environment changes due to seasonal effects, response to the news, dynamics of the market, etc. When $Z_{n}$ is a function of the state of a Markov chain, stochastic difference Equation (1) is a formal analogue of the Langevin equation with regime switches, which was studied in [18]. The notion of regime shifts or regime switches traces 
back to $[19,20]$, where it was proposed in order to explain the cyclical feature of certain macroeconomic variables.

In this paper we consider $\left(Z_{n}\right)_{n \in \mathbb{Z}}$ that belong to the following class of random processes:

Definition 2 ([21]). A C-chain is a stationary random process $\left(Z_{n}\right)_{n \in \mathbb{Z}}$ taking values in a finite set (alphabet)

$D$, such that the following holds:

i) For any $i_{1} i_{2}, \cdots, i_{n} \in D$, $P\left(Z_{1}=i_{1} Z_{2}=i_{2}, \cdots, Z_{n}=i_{n}\right)>0$.

ii) For any $i_{0} \in D$ and any sequence $\left(i_{n}\right)_{n \in \mathbb{N}} \in D^{\mathbb{N}}$, the following limit exists:

$$
\begin{gathered}
\lim _{n \rightarrow \infty} P\left(Z_{0}=i_{0} \mid Z_{-k}=i_{k}, 1 \leq k \leq n\right) \\
P\left(Z_{0}=i_{0} \mid Z_{-k}=i_{k}, k \geq 1\right)
\end{gathered}
$$

where the right-hand side is a regular version of the conditional probabilities.

iii) Let

$\gamma_{n}=\sup \left\{\left|\frac{P\left(Z_{0}=i_{0} \mid Z_{-k}=i_{k}, k \geq 1\right)}{P\left(Z_{0}=i_{0} \mid Z_{-k}=j_{k}, k \geq 1\right)}-1\right|: i_{k}=j_{k}, k \leq n\right\}$.

Then, $\limsup _{n \rightarrow \infty} \frac{\gamma_{n}}{n}<0$.

$C$-chains form an important subclass of chains with complete connections/chains of in-finite order [22-24]. They can be described as exponentially mixing full shifts, and alternatively defined as an essentially unique random process with a given transition function ( $g$-measure) $P\left(X_{0}=i_{0} \mid X_{k}=i_{k}, k<0\right) \quad$ [25]. Stationary distributions of these processes are Gibbs states in the sense of Bowen $[21,26]$. For any $C$-chain $\left(Z_{n}\right)_{n \in \mathbb{Z}}$ there exists a Markovian representation [21,25], that is a stationary irreducible Markov chain $\left(Y_{n}\right)_{n \in \mathbb{Z}}$ in a countable state space and a function $\zeta: S \rightarrow D$ such that $\left(Z_{n}\right)_{n \in \mathbb{Z}}={ }_{D}\left(\zeta\left(Y_{n}\right)\right)_{n \in \mathbb{Z}}$, where ${ }{ }_{D}$ means equivalence of distributions. Chains of infinite order are well-suited for modeling of long range-dependence with fading memory, and in this sense constitute a natural generalization of finite-state Markov chains [24,27-30].

We will further assume that the vectors $Q_{n, i}$ are multivariate regularly varying. Recall that, for $\alpha \in \mathbb{R}$, a function $f: \mathbb{R} \rightarrow \mathbb{R}$ is said to be regularly varying of index $\alpha$ if $f(t)=t^{\alpha} L(t)$ for some function $L: \mathbb{R} \rightarrow \mathbb{R}$ such that $L(\lambda t) \sim L(t)$ for any positive real $\lambda>0$ (i.e., $L$ is a slowly varying function). Let $\overline{\mathbb{R}}_{0}^{d}:=[-\infty,+\infty]^{d} /\{0\}$.

Definition 3 ([31]). A random vector $Q \in \mathbb{R}^{\mathrm{d}}$ is regularly varying with index $\alpha>0$ if there exist a function $b: \mathbb{R} \rightarrow \mathbb{R}$ regularly varying with index $1 / \alpha$ and a Radon measure $\mu$ in the space $\overline{\mathbb{R}}_{0}^{\mathrm{d}}$ such that $n P\left(b_{n}^{-1} Q \in \cdot\right) \rightarrow_{v} \mu(\cdot)$, as $n \rightarrow \infty$, where $\rightarrow_{v}$ denotes the vague convergence and $b_{n}:=b(n)$.

We denote by $\mathfrak{R}_{\mathrm{d}, \alpha, b}$ the set of all $d$-vectors regularly varying with index $\alpha$, associated with function $b$.

The corresponding limiting measure $v$ is called the measure of regular variation associated with $Q$.

We next summarize our assumptions on the coefficients $Q_{n}$ and $M$. Let $\|Q\|:=\max _{1 \leq i \leq d}|Q(i)|$ and $\|M\|:=\sup _{q \in \mathbb{R}^{\mathrm{d}}}\{\|M q\|:\|q\|=1\}$ for, respectively, a vector $Q=Q(Q(1), Q(2), \cdots, Q(\mathrm{~d})) \in \mathbb{R}^{\mathrm{d}}$ and a $\mathrm{d} \times \mathrm{d}$ matrix $M$.

Assumption 1. Let $\left(Z_{n}\right)_{n \in \mathbb{Z}}$ be a stationary $C$-chain defined on a finite state space $D$, and suppose that $\left(Q_{n}\right)_{n \in \mathbb{Z}}$ is induced by $\left(Z_{n}\right)_{n \in \mathbb{Z}}$. Assume in addition that:

A1) $E\left[\log ^{+}\left\|Q_{0}\right\|\right]<+\infty$, where $x^{+}:=\max \{x, 0\}$ for $x \in \mathbb{R}$.

A2) The spectral radius $\lim _{n \rightarrow \infty} \sqrt[n]{\left\|M^{n}\right\|}$ is strictly between zero and one.

A3) There exist a constant $\alpha>0$ and a regularly varying function $b$ with index $\alpha^{-1}$ such that for all $i \in D, Q_{0 . i} \in \mathfrak{R}_{\mathrm{d}, \alpha, b}$ with associated measure of regular variation $\mu_{i}$.

\section{Statement of Results}

For any (random) initial vector $V_{0}$, the series $V_{n}$ converges in distribution, as $n \rightarrow \infty$, to

$$
V:=\sum_{k=0}^{\infty} M^{k} Q_{-k},
$$

which is the unique initial value making $\left(V_{n}\right)_{n \geq 0}$ into a stationary sequence [32]. The following result, whose proof is omitted, is a "Gibssian" version of a "Markovian" [16, Theorem 1]. The claim can be established following the line of argument in [16] nearly verbatim, exploiting the Markov representation of C-chains obtained in [21].

Theorem 1. Let Assumption 1 hold. Then $V \in \mathfrak{R}_{\mathrm{d}, \alpha, b}$ with associated measure of regular variation

$$
\mu_{V}(\cdot)=\sum_{k=0}^{\infty} E\left[\mu_{Q_{0}} \circ\left(M^{k}\right)^{-1}(\cdot)\right],
$$

where $\mu \circ \prod^{-1}(\cdot)$ stands for $\mu\left(\left\{x \in \mathbb{R}^{\mathrm{d}}: \prod x \in \cdot\right\}\right)$ and $\mu_{Q_{0}}(\cdot):=\sum_{i \in D} P\left(Z_{0}=i\right) \mu_{i}(\cdot)$.

In a slightly more general setting, the existence of the limiting velocity suggests the following law of large numbers, whose short proof is included in Section 3.1. 
Theorem 2. Let Assumption 1 hold with A3) being replaced by the condition $E\left[\left\|Q_{0}\right\|\right]<\infty$. Then 1

$$
\lim _{n \rightarrow \infty} \frac{X_{n}}{n}=E[V]=(I-M)^{-1} E\left[Q_{0}\right] \text {, a.s. }
$$

Let $\left(\bar{Q}_{n}\right)_{n \in \mathbb{Z}}$ denote independent copies of $Q_{0}$, and let be $\left(A_{n}\right)_{n \in \mathbb{Z}}$ a sequence of vectors such that the sequence of processes

$$
\bar{S}_{t}^{(n)}=b_{n}^{-1}\left(\sum_{k=1}^{[n t]} \bar{Q}_{k}-A_{[n t]}\right), t>0,
$$

converges in law as $n \rightarrow \infty$ in the Skorokhod space $D\left(\mathbb{R}_{+}, \mathbb{R}^{d}\right)$ to a Lévy process $\xi_{t}^{(\alpha, \mu)}, t>0, \mu=\left(\mu_{i}\right)_{i \in D}$, where $\mu_{i}$ are introduced in A3) with stationary independent increments, $\xi_{0}^{(\alpha, \mu)}=0$, and $\xi_{1}^{(\alpha, \mu)}$ being distributed according to a stable law of index $\alpha$ whose domain of attraction includes $Q_{0}$. For an explicit form of the centering sequence $A_{n}$ and the characteristic function of $\xi_{1}^{(\alpha, \mu)}$ see, for instance, [33] or [34]. Remark that one can set $A_{n}=0$ if $\alpha<1$ and $A_{n}=n E\left[Q_{0}\right]$ if $\alpha>1$. For each $n \in \mathbb{N}$, define a process $S^{(n)}$ in $D\left(\mathbb{R}_{+}, \mathbb{R}^{\mathrm{d}}\right)$ by setting

$$
S_{t}^{(n)}=b_{n}^{-1}\left(X_{[n t]}-(I-M)^{-1} A_{[n t]}\right), t>0 .
$$

Theorem 3. Let Assumption 1 hold with $\alpha \in(0,2)$. Then the sequence of processes $S^{(n)}$ converges weakly in $D\left(\mathbb{R}_{+}, \mathbb{R}^{\mathrm{d}}\right)$, as $n \rightarrow \infty$, to $(I-M)^{-1} \xi_{t}^{(\alpha, \mu)}$.

It follows from Definition 3 (see, for instance, [31]) that if $Q \in \mathfrak{R}_{\mathrm{d}, \alpha, b}$ then the following limit exists for any vector $x \in \mathbb{R}^{\mathrm{d}}$ :

$$
w(x):=\lim _{n \rightarrow \infty} n P\left(Q \cdot x \geq b_{n}\right)
$$

Theorem 4. Assume that the conditions of Theorem 3 hold. If $\alpha=1$ assume in addition that the law of $Q_{0 . i}$ is symmetric for any $i \in D$. Let $w(x)$ be defined by Equation (4) with $Q=(I-M)^{-1} Q_{0}$. Then, for any $x \in \mathbb{R}^{\mathrm{d}}$ such that $w(x)>0$, we have

$$
\limsup _{n \rightarrow \infty} \frac{\left(X_{n}-A_{n}\right) \cdot x}{b_{n}(\ln n)^{1 / \alpha+\varepsilon}}=\left\{\begin{array}{l}
0 \text { if } \varepsilon>0, \\
\infty \text { if } \varepsilon<0,
\end{array} \quad\right. \text { a.s. }
$$

In particular,

$$
\limsup _{n \rightarrow \infty}\left(\frac{\left(X_{n}-A_{n}\right) \cdot x}{b_{n}}\right)^{1 / \ln \ln n}=\mathrm{e}^{1 / \alpha} \text {, a.s. }
$$

If either Assumption 1 holds with $\alpha=2$ or $E\left[\left\|Q_{0}\right\|^{2}\right]<\infty$ is assumed instead of A3), then, in view of Equation (6), a Gaussian counterpart of Theorem 3 can be obtained as a direct consequence of general CLTs for uniformly mixing sequences (see, for instance, [35,
Theorem 20.1] and [36, Corollary 2]) applied to the sequence $\left(Q_{n}\right)_{n \in \mathbb{Z}}$. If $E\left[\left\|Q_{0}\right\|^{2}\right]<\infty$, then a law of iterated logarithm in the usual form follows from Equation (5) and, for instance, [37, Theorem 5] applied to the sequence $\left(Q_{n}\right)_{n \in \mathbb{Z}}$.

We remark that in the case of i.i.d. additive component $Q_{n}$, similar to our results are obtained in [7] for a more general than Equation (1) mapping $V_{n+1}=\Phi\left(V_{n}\right)$.

\section{Proofs}

\subsection{Proof of Theorem 2}

It follows from the definition of the random walk $X_{n}$ and Equation (1) that

$$
\begin{aligned}
& X_{n} \\
= & \sum_{k=1}^{n}\left(M^{k} V_{0}+\sum_{t=1}^{k} M^{k-t} V_{t}\right) \\
= & \sum_{k=1}^{n} M^{k} V_{0}+\sum_{t=1}^{n}\left(\sum_{k=t}^{\infty} M^{k-t}-\sum_{k=n+1}^{\infty} M^{k-t}\right) Q_{t} \\
= & \sum_{k=1}^{n} M^{k} V_{0}+(I-M)^{-1} \sum_{t=1}^{n} Q_{t} \\
+ & (I-M)^{-1} \sum_{t=1}^{n} M^{n+1-t} Q_{t} .
\end{aligned}
$$

Note that $E\left[\left\|Q_{0}\right\|\right]<\infty$ implies

$$
\sum_{n=1}^{\infty} P\left(\left\|Q_{0}\right\|>n \varepsilon\right)<\infty .
$$

It follows then from the Borel-Cantelli lemma that

$$
\lim _{n \rightarrow \infty} \frac{1}{n}\left\|\sum_{t=1}^{\infty}\right\| M^{n+1-t} V_{t}\|<\infty\|=0, \quad \text { a.s. }
$$

Furthermore, we have

$$
P\left(\sum_{k=1}^{\infty}\left\|M^{k} V_{0}\right\|<\infty\right)=1 .
$$

Thus the law of large numbers for $X_{n}$ follows from the ergodic theorem applied to the sequence $\left(Q_{n}\right)_{n \in \mathbb{Z}}$.

\subsection{Proof of Theorem 3}

Only the second term in the right-most side of Equation (5) contributes to the asymptotic behavior of $X_{n}$. The proof rests on the application of Corollary 5.9 in [34] to the partial sums $\sum_{t=1}^{n} Q_{t}$. In view of condition iii) in

Definition 2 and the decomposition shown in Equation (6), we only need to verify that the following "local dependence" condition (which is condition (5.13) in [34]) holds for the sequence $\left(Q_{n}\right)_{n \in \mathbb{Z}}$ :

$$
n P\left(\left\|Q_{0}\right\|>b_{n} \varepsilon,\left\|Q_{j}\right\|>b_{n} \varepsilon\right) \rightarrow_{n \rightarrow \infty} 0, \forall \varepsilon>0, \forall j \in \mathbb{N}
$$

The above convergence to zero follows from the mix- 
ing condition iii) in Definition 2 and the regular variation, as $t$ goes to infinity, of the marginal distribution tail $P\left(\left\|Q_{0}\right\|>t\right)=\sum P\left(Z_{0}=i\right) \cdot P\left(\left\|Q_{j, i}\right\|>t\right)$.

\subsection{Proof of Theorem 4}

For $i \in D$, let $k_{n}(i)$ be the number of occurrences of $i$ in the set $\left\{Z_{1}, Z_{2}, \cdots, Z_{n}\right\}$. That is,

$$
k_{n}(i)=\sum_{j=1}^{n} \mathrm{I}_{\left\{Z_{j}=i\right\}} .
$$

Define recursively $T_{i}(0)=0$ and

$$
T_{i}(j)=\inf \left\{k>T_{i}(j-1): Z_{k}=i\right\}
$$

(with the usual convention that the greatest lower bound over an empty set is equal to infinity). For $i \in D, n \in \mathbb{N}$, let

$$
S_{n, i}=\sum_{j=1}^{n}(I-M)^{-1} Q_{T_{i}(j), i}-C_{n, i},
$$

where

$$
C_{n, i}:=\left\{\begin{array}{l}
0 \text { if } \alpha \leq 1 \\
n \cdot E\left[(I-M)^{-1} Q_{0, i}\right] \text { if } \alpha \in(1,2)
\end{array} .\right.
$$

Denote

$$
\tilde{X}_{n}:=\sum_{i \in D} S_{k_{n}(i), i} .
$$

Further, for each $n \in \mathbb{N}$ let $R_{n}=0$ if $\alpha \leq 1$, whereas if $\alpha>1$ let

$$
R_{n}=\sum_{i \in D} E\left[(I-M)^{-1} Q_{0, i} \cdot\left\{k_{n}(i)-n P\left(Z_{0}=i\right)\right\}\right] .
$$

Then $\tilde{X}_{n}-R_{n}=(I-M)^{-1} \sum_{j=1}^{n} Q_{j}-A_{n}$, and hence

$$
X_{n}-(I-M)^{-1} \sum_{j=1}^{n} Q_{j}=\left(X_{n}-A_{n}\right)-\left(\tilde{X}_{n}-R_{n}\right) \text {. }
$$

It follows from the decomposition given by Equation (6) along with the Borel-Cantelli lemma that for any $x \in \mathbb{R}^{\mathrm{d}}$,

$$
\lim _{n \rightarrow \infty} \frac{\left(X_{n}-A_{n}\right) \cdot x-\left(\tilde{X}_{n}-R_{n}\right) \cdot x}{b_{n}(\ln n)^{1 / \alpha+\varepsilon}}=0, \quad \text { a.s. }
$$

Let $\mathfrak{I}_{n}(i)=\mathrm{I}_{\left\{Z_{n}=i\right\}}-P\left(Z_{0}=i\right)$. Then

$$
k_{n}(i)-n P\left(Z_{0}=i\right)=\sum_{j=1}^{n} \mathfrak{J}_{j}(i) .
$$

It follows, for instance, from Theorem 5 in [37] that if $\alpha>1$ then for any $x \in \mathbb{R}^{\mathrm{d}}$, the following limit exists and the identity holds with probability one:

$$
\begin{aligned}
& \limsup \sup _{n \rightarrow \infty} \frac{R_{n} \cdot x}{\sqrt{2 n \ln \ln n}} \\
\leq & \sum_{i \in D} E\left[\left|(I-M)^{-1} Q_{0, i} \cdot x\right|\right] \\
\times & \limsup _{n \rightarrow \infty} \frac{\sum_{j=1}^{n} \Im_{j}(i) \cdot x}{\sqrt{2 n \ln \ln n}}<\infty .
\end{aligned}
$$

Therefore (since $b_{n}$ is regularly varying with index $\alpha<2$ ), in order to complete the proof Theorem 4 it suffices to show that for any $x \in \mathbb{R}^{\mathrm{d}}$ that satisfies the condition $w(x)>0$ of the theorem, we have

$$
\limsup _{n \rightarrow \infty} \frac{\tilde{X}_{n} \cdot x}{b_{n}(\ln n)^{1 / \alpha+\varepsilon}}=\left\{\begin{array}{l}
0 \text { if } \varepsilon>0, \\
\infty \text { if } \varepsilon<0,
\end{array}\right. \text { a.s. }
$$

We first observe that by the law of iterated logarithm for heavy-tailed i.i.d. sequences (see Theorems 1.6.6 and 3.9 .1 in [33]),

$$
\limsup _{n \rightarrow \infty} \frac{S_{k_{n}(i), i} \cdot x}{b_{k_{n}(i)}\left(\ln k_{n}(i)\right)^{1 / \alpha+\varepsilon}}=0, \quad \text { a.s. }
$$

for any $i \in D, \quad x \in \mathbb{R}^{\mathrm{d}}$, and $\varepsilon>0$. Since by the ergodic theorem,

$$
\lim _{n \rightarrow \infty} k_{n}(i) / n=P\left(Z_{0}=i\right) \in(0, \infty), \quad \text { a.s. }
$$

this yields

and hence

$$
\limsup _{n \rightarrow \infty} \frac{S_{k_{n}(i)} \cdot x}{b_{n}(\ln n)^{1 / \alpha+\varepsilon}}=0, \quad \text { a.s., }
$$

$$
\limsup _{n \rightarrow \infty} \frac{\tilde{X}_{n} \cdot x}{b_{n}(\ln n)^{1 / \alpha+\varepsilon}}=0, \quad \text { a.s. }
$$

On the other hand, if $\varepsilon<0$, Theorem 3.9.1 in [33] implies that for any $i \in D$ and any $x \in \mathbb{R}^{\mathrm{d}}$ such that $w(x)>0$, we have

$$
\limsup _{n \rightarrow \infty} \frac{S_{k_{n}(i), i} \cdot x}{b_{k_{n}(i)}\left(\ln k_{n}(i)\right)^{1 / \alpha+\varepsilon}}=\infty, \quad \text { a.s. }
$$

To conclude the proof of the theorem it thus remains to show that for any $i, j \in D$, any $x \in \mathbb{R}^{\mathrm{d}}$, and all $\delta \in(1 /(2 \alpha), 1 / \alpha)$,

$$
P\left(E_{i, x}(n) \cap E_{j, x}(n) \text { i.o. }\right)=0,
$$

where, for $i \in D$, the events $E_{i, x}(n)$ are defined as follows:

$$
E_{i, x}(n):=\left\{\left|S_{k_{n}(i), i} \cdot x\right|>b_{n}(\ln n)^{\delta}\right\} .
$$

For $i \in D$, let $\xi_{i}=2 \cdot P\left(Z_{0}=i\right)$ and define

$$
G_{n, i}:=\left\{\max _{1 \leq m \leq n \xi_{i}}\left|S_{k_{n}(i), i} \cdot x\right|>b_{n}(\ln n)^{\delta}\right\} .
$$

Then

$$
\begin{aligned}
& P\left(E_{i, x}(n) \cap E_{j, x}(n)\right) \\
\leq & P\left(G_{n, i} \cap G_{n, j}\right)+P\left(k_{n}(i)>n \xi_{i}\right) \\
+ & P\left(k_{n}(j)>n \xi_{j}\right) \\
= & P\left(G_{n, i}\right) \cdot P\left(G_{n, j}\right)+P\left(k_{n}(i)>n \xi_{i}\right) \\
+ & P\left(k_{n}(j)>n \xi_{j}\right)
\end{aligned}
$$


The Ruelle-Perron-Frobenius theorem (see [26]) implies that the sequence $k_{n} / n$ satisfies the large deviation principle (by the Gärtner-Ellis theorem), and hence $P\left(k_{n}(i)>n \xi_{i}\right)<C_{i} \mathrm{e}^{-n \lambda_{i}}$ for some constants $C_{i}>0$ and $\lambda_{i}>0$. Furthermore, for any $A>0, n_{k}=\left[A^{k}\right]$, and $\beta \in(0, \alpha)$ there exists a constant $C=C(A, \beta)$ such that (see [33, p. 177]), $P\left(E_{n_{k}, i}\right) \leq C k^{-\delta \beta}$. Therefore, since $\delta>2 / \alpha$, we can choose $\beta \in(0, \alpha)$ such that $P\left(E_{n_{k}, i}\right) \cdot P\left(E_{n_{k}, j}\right) \leq C_{0} k^{-\gamma}$, with suitable $C_{0}>0$ and $\gamma>1$. A standard argument using the Borel-Cantelli lemma imply then the identity in Equation (7). $\square$

\section{REFERENCES}

[1] W. T. Coffey, Yu. P. Kalmykov and J. T. Waldron, "The Langevin Equation: With Applications to Stochastic Problems in Physics, Chemistry and Electrical Engineering," 2nd Edition, World Scientific Series in Contemporary Chemical Physics, Vol. 14, World Scientific Publishing Company, Singapore City, 2004.

[2] N. G. Van Kampen, "Stochastic Processes in Physics and Chemistry," 3th Edition, North-Holland Personal Library, Amsterdam, 2003.

[3] O. E. Barndorff-Nielsen and N. Shephard, "Non-Gaussian Ornstein-Uhlenbeck-Based Models and Some of Their Uses in Financial Economics (with Discussion)," Journal of the Royal Statistical Society: Series B (Statistical Methodology), Vol. 63, No. 2, 2001, pp. 167-241. doi:10.1111/1467-9868.00282

[4] S. C. Kou and X. S. Xie, "Generalized Langevin Equation with Fractional Gaussian Noise: Sub-Diffusion within a Single Protein Molecule," Physical Review Letters, Vol. 93, 2004, Article ID: 18.

[5] D. Buraczewski, E. Damek and M. Mirek, "Asymptotics of Stationary Solutions of Multivariate Stochastic Recursions with Heavy Tailed Inputs and Related Limit Theorems," Stochastic Processes and Their Applications, Vol. 122, No. 1, 2012, pp. 42-67. doi:10.1016/j.spa.2011.10.010

[6] H. Larralde, "A First Passage Time Distribution for a Discrete Version of the Ornstein-Uhlenbeck Process," Journal of Physics A, Vol. 37, No. 12, 2004, pp. 37593767. doi:10.1088/0305-4470/37/12/003

[7] H. Larralde, "Statistical Properties of a Discrete Version of the Ornstein-Uhlenbeck Process," Physical Review E, Vol. 69, 2004, Article ID: 027102.

[8] E. Renshaw, "The Discrete Uhlenbeck-Ornstein Process," Journal of Applied Probability, Vol. 24, No. 4, 1987, pp. 908-917. doi: $10.2307 / 3214215$

[9] M. Lefebvre and J.-L. Guilbault, "First Hitting Place Probabilities for a Discrete Version of the OrnsteinUhlenbeck Process," International Journal of Mathematics and Mathematical Sciences, Vol. 2009, 2009, Article ID: 909835

[10] A. Novikov and N. Kordzakhia, "Martingales and First Passage Times of AR(1) Sequences," Stochastics, Vol. 80,
No. 2-3, 2008, pp. 197-210. doi:10.1080/17442500701840885

[11] P. Embrechts and C. M. Goldie, "Perpetuities and Random Equations," Proceedings of 5th Prague Symposium, Physica, Heidelberg, 1993, pp. 75-86.

[12] R. F. Engle, "ARCH. Selected Readings," Oxford University Press, Oxford, 1995.

[13] S. T. Rachev and G. Samorodnitsky, "Limit Laws for a Stochastic Process and Random Recursion Arising in Probabilistic Modelling," Advances in Applied Probability, Vol. 27, No. 1, 1995, pp. 185-202.

[14] W. Vervaat, "On a Stochastic Difference Equations and a Representation of Non-Negative Infinitely Divisible Random Variables," Advances in Applied Probability, Vol. 11, No. 4, 1979, pp. 750-783. doi:10.2307/1426858

[15] Y. Ephraim and N. Merhav, "Hidden Markov Processes," IEEE Transactions on Information Theory, Vol. 48, No. 6, 2002, pp. 1518-1569. doi:10.1109/TIT.2002.1003838

[16] D. Hay, R. Rastegar and A. Roitershtein, "Multivariate Linear Recursions with Markov-Dependent Coefficients," Journal of Multivariate Analysis, Vol. 102, No. 3, 2011, pp. 521-527. doi:10.1016/j.jmva.2010.10.011

[17] R. Stelzer, "Multivariate Markov-Switching ARMA Processes with Regularly Varying Noise," Journal of Multivariate Analysis, Vol. 99, No. 6, 2008, pp. 1177-1190. doi:10.1016/j.jmva.2007.07.001

[18] P. Eloe, R. H. Liu, M. Yatsuki, G. Yin and Q. Zhang, "Optimal Selling Rules in a Regime-Switching Exponential Gaussian Diffusion Model," SIAM Journal of Applied Mathematics, Vol. 69, No. 3, 2008, pp. 810-829. doi:10.1137/060652671

[19] E. F. Fama, "The Behavior of Stock Market Prices," Journal of Business, Vol. 38, No. 1, 1965, pp. 34-105. doi:10.1086/294743

[20] J. D. Hamilton, "A New Approach to the Economic Analysis of Non-Stationary Time Series and the Business Cycle," Econometrica, Vol. 57, No. 2, 1989, pp. 357-384. doi:10.2307/1912559

[21] S. Lalley, "Regenerative Representation for One-Dimensional Gibbs States," Annals of Probability, Vol. 14, No. 4, 1986, pp. 1262-1271. doi:10.1214/aop/1176992367

[22] R. Fernández and G. Maillard, "Chains with Complete Connections and One-Dimensional Gibbs Measures," Electronic Journal of Probability, Vol. 9, 2004, pp. 145176. doi:10.1214/EJP.v9-149

[23] R. Fernández and G. Maillard, "Chains with Complete Connections: General Theory, Uniqueness, Loss of Memory and Mixing Properties," Journal of Statistical Physics, Vol. 118, No. 3-4, 2005, pp. 555-588. doi:10.1007/s10955-004-8821-5

[24] M. Iosifescu and S. Grigorescu, "Dependence with Complete Connections and Its Applications," Cambridge Tracts in Mathematics, Vol. 96, Cambridge University Press, Cambridge, 2009.

[25] H. Berbee, "Chains with Infinite Connections: Uniqueness and Markov Representation," Probability Theory and Related Fields, Vol. 76, No. 2, 1987, pp. 243-253. doi:10.1007/BF00319986 
[26] R. Bowen, "Equillibrium States and the Ergodic Theory of Anosov Diffeomorthisms," Lecture Notes in Mathematics, Vol. 470, Springer, Berlin, 1975.

[27] F. Comets, R. Fernández and P. A. Ferrari, "Processes with Long Memory: Regenerative Construction and Perfect Simulation," Annals of Applied Probability, Vol. 12, No. 3, 2002, pp. 921-943. doi:10.1214/aoap/1031863175

[28] A. Galves and E. Löcherbach, "Stochastic Chains with Memory of Variable Length," TICSP Series, Vol. 38, 2008, pp. 117-133.

[29] J. Rissanen, "A Universal Data Compression System," IEEE Transactional on Information Theory, Vol. 29, No. 5, 1983, pp. 656-664. doi:10.1109/TIT.1983.1056741

[30] R. Vilela Mendes, R. Lima and T. Araújo, "A ProcessReconstruction Analysis of Market Fluctuations," International Journal of Theoretical Applied Finance, Vol. 5, No. 8, 2002, pp. 797-821. doi:10.1142/S0219024902001730

[31] S. I. Resnick, "On the Foundations of Multivariate Heavy Tail Analysis," Journal of Applied Probability, Vol. 41, 2004, pp. 191-212. doi:10.1239/jap/1082552199

[32] A. Brandt, "The Stochastic Equation $Y_{n+1}=A_{n} Y_{n}+B_{n}$ with
Stationary Coefficients," Advances in Applied Probability, Vol. 18, No. 1, 1986, pp. 211-220. doi:10.2307/1427243

[33] A. A. Borovkov and K. A. Borovkov, "Asymptotic Analysis of Random Walks: Heavy-Tailed Distributions," Encyclopedia of Mathematics and Its Applications, Vol. 118, Cambridge University Press, Cambridge, 2008.

[34] M. Kobus, "Generalized Poisson Distributions as Limits for Sums of Arrays of Dependent Random Vectors," Journal of Multivariate Analysis, Vol. 52, No. 2, 1995, pp. 199-244. doi:10.1006/jmva.1995.1011

[35] P. Billingsley, "Convergence of Probability Measures," John Wiley \& Sons, New York, 1968.

[36] Z. S. Szewczak, "A Central Limit Theorem for Strictly Stationary Sequences in Terms of Slow Variation in the Limit," Probability and Mathematical Statistics, Vol. 18, No. 2, 1998, pp. 359-368.

[37] H. Oodaria and K. Yoshihara, "The Law of the Iterated Logarithm for Stationary Processes Satisfying Mixing Conditions," Kodai Mathematical Seminar Reports, Vol. 23, No. 3, 1971, pp. 311-334. doi: $10.2996 / \mathrm{kmj} / 1138846370$ 